Article

\title{
Synthesis and Activity of Novel Acylthiourea with Hydantoin
}

\author{
Jintao Han ${ }^{1}$, Hongbo Dong ${ }^{1}$, Zhihong Xu ${ }^{1,2}$, Jinmin Wang ${ }^{1}$ and Mingan Wang ${ }^{1, *}$ \\ 1 Department of Applied Chemistry, China Agricultural University, Beijing 100193, China; \\ E-Mails: hanjint321@163.com (J.H.); bloodwhenseeme@163.com (H.D.); \\ x_u_78@sina.com (Z.X.); wangjinmin@126.com (J.W.) \\ 2 College of Agricuture, Yangtze University, Jingzhou 434025, Hubei, China \\ * Author to whom correspondence should be addressed; E-Mail: wangma@cau.edu.cn; \\ Tel.: +86-10-6273-4093; Fax: +86-10-6273-7732.
}

Received: 23 July 2013; in revised form: 29 August 2013 / Accepted: 18 September 2013 /

Published: 26 September 2013

\begin{abstract}
The 41 novel acylthiourea derivatives with hydantoin were synthesized in moderate to excellent yields by using 5-(4-aminophenyl)- and 5-(4-aminobenzyl)hydantoin or 5-(4-aminobenzyl)-thiohydantoin as raw materials and characterized by IR, ${ }^{1} \mathrm{H}$ NMR spectra and elementary analysis. The preliminary bioassay showed that these compounds exhibit certain selectively herbicidal activities with the $91 \%, 94 \%$ and $87 \%$ inhibition rates of $7 \mathbf{7}, \mathbf{8 0}$ and $\mathbf{8 p}$ against $B$. campestris, $100 \%, 100 \%$ and $95 \%$ efficacy against $B$. campestris in a greenhouse test, respectively. $\mathbf{7 a}, \mathbf{7 b}, \mathbf{7} \mathbf{c}$ and $\mathbf{7 d}$ exhibited $74 \%$, $79 \%, 79 \%$ and $71 \%$ inhibition rates against $F$. oxysporum, respectively.
\end{abstract}

Keywords: acylthiourea; hydantoin; thiohydantoin; herbicidal activity; fungicidal activity

\section{Introduction}

Hydantoin and thiohydantion are important core moiety in the design and synthesis of active molecules as well as natural products; these derivatives have not only been used in medicinal chemistry as anti-HSV, antidiabetic, HDL-cholesterol modulators, but also used as fungicides and herbicides in agrochemical research [1-6]. In addition, these derivatives are very useful building blocks for the synthesis of various heterocycles such as 5-arylidene derivatives of imidazoline-4-one, imidazothiazine, diazinone, and diazepinone [7-9]. In our laboratory, several types of hydantoin and thiohydantioin derivatives $(\mathbf{1}, \mathbf{2}, \mathbf{3})$ were synthesized, and we showed that some of them exhibit good 
herbicidal, fungicidal and insecticidal activities [10-13]. Acylthioureas are the key structural motifs of numerous compounds that displayed a wide range of biological activity such as antimicrobial, antipathogenic, insecticidal, fungicidal, antitumor activities and influenza virus inhibitors [14-21]. Acylthioureas are widely used as the building blocks for the synthesis of various heterocycles such as thiazolidene-2-imine, imidazole-2-thione and 2-thioxo-4-imidazolidinone [22-33]. We could not find acylthiourea derivatives containing hydantoin and thiohydantion heterocycles in references. Continuing effort of our ongoing project aimed at looking for novel biologically active hydantoin and thiohydantion heterocyclic compounds [10-13], herein, we report a new type of acylthiourea derivatives containing hydantoin and thiohydantion heterocycles (Scheme 1) and their biological activities.

Scheme 1. The synthetic route of new acylthiourea derivatives.<smiles>[X]C1NC(=O)C(CCCC)N1</smiles>

1

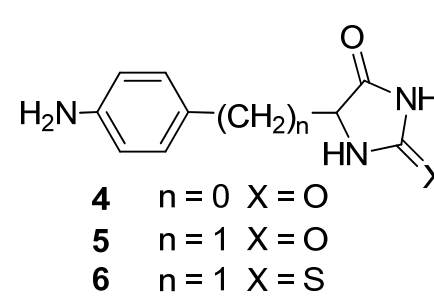<smiles>[X]C1NC(=O)C(CCCC)N1</smiles>

2<smiles>[X]C1NC(=O)C(CCCC)N1</smiles>

3

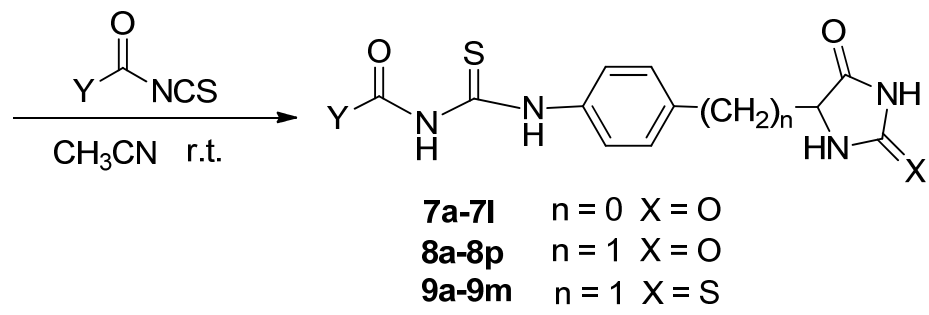
7a, 8a, 9a $\mathrm{Y}=\mathrm{C}_{6} \mathrm{H}_{5}$
7b, 8b, 9b $\mathrm{Y}=p-\mathrm{FC}_{6} \mathrm{H}_{4}$
7c, 8c, 9c $\mathrm{Y}=p-\mathrm{CIC}_{6} \mathrm{H}_{4}$
7d, 8d, 9d $\mathrm{Y}=p-\mathrm{CH}_{3} \mathrm{C}_{6} \mathrm{H}_{4}$
8 e, 9e $\mathrm{Y}=p-\mathrm{CH}_{3} \mathrm{OC}_{6} \mathrm{H}_{4}$
8f, 9f $\mathrm{Y}=p-\mathrm{NO}_{2} \mathrm{C}_{6} \mathrm{H}_{4}$
7e, 8g, 9g $\mathrm{Y}=\mathrm{O}-\mathrm{CH}_{3} \mathrm{C}_{6} \mathrm{H}_{4}$
7f, 8h, 9h $\mathrm{Y}=\mathrm{o}-\mathrm{ClC}_{6} \mathrm{H}_{4}$
8i, 9i $\mathrm{Y}=\mathrm{O}-\mathrm{CH}_{3} \mathrm{OC}_{6} \mathrm{H}_{4}$
7g, 8j, 9j $\mathrm{Y}=\mathrm{o}-\mathrm{NO}_{2} \mathrm{C}_{6} \mathrm{H}_{4}$
7h, 8k, 9k $\mathrm{Y}=m-\mathrm{CH}_{3} \mathrm{C}_{6} \mathrm{H}_{4}$
7i, 8I, 9l $\mathrm{Y}=m-\mathrm{NO}_{2} \mathrm{C}_{6} \mathrm{H}_{4}$
7j, 8m, 9m $\mathrm{Y}=2-\mathrm{C}_{4} \mathrm{H}_{3} \mathrm{~S}$
7k, 8n $\mathrm{Y}=2-\mathrm{CIC}_{5} \mathrm{H}_{3} \mathrm{~N}$
7I, $80 \mathrm{Y}=2,4-\mathrm{Cl}_{2} \mathrm{C}_{6} \mathrm{H}_{3} \mathrm{OCH}_{2}$
$8 p \mathrm{Y}=4-\mathrm{ClC}_{6} \mathrm{H}_{4} \mathrm{OCH}_{2}$

\section{Results and Discussion}

In our laboratory, we synthesized 5-aryl derivatives of hydantoin and thiohydantoin to develop the novel inhibitor of Adenylosuccinate Synthetase (AdSS) [10-13], which plays a key role in the two-step conversion of IMP to AMP in the de novo pathway of purine biosynthesis [34-36]. Some of the esters 1 were tested and showed strong herbicidal activities against Zea mays, Triticum aestivum and Arabidopsis thaliana, the further greenhouse test showed that compounds have $60 \%, 50 \%$ and $50 \%$ efficacy against Stellaria media, Echinochloa crus-galli and Setaria viridis at the dosage of $1000 \mathrm{~g} / \mathrm{ha}$ when used as a pre-emergence treatment, respectively [13]. After that, the thiophosphates 2 and phosphoramidates were found to show weak herbicidal activities against Brasica campestris and Echinochloa crus-galli, while one of them exhibited excellent insecticidal activities against Myzus Persicae [11,12]. Then, phosphoramides 3 were further tested and showed increasing herbicidal activities against Brasica campestris as well as insecticidal activities against Myzus Persicae [37]. These results indicated that 5-(4-aminophenyl)-hydantoin, 5-(4-aminobenzyl)-hydantoin and 5-(4-aminobenzyl)-thiohydantoin were important moiety for these active compounds. On the other 
hand, acylthiourea derivatives have been widely used and synthesized in medicinal chemistry and agrochemical research [14-21] in recent years. Based on these characters, we combined the containing-hydantoin amino derivatives and acylthioureas into a molecule and designed the novel acylthiourea derivatives containing hydantoin and thiohydantoin heterocycles $(\mathbf{7}, \mathbf{8}, \mathbf{9})$. The synthesis was carried out by the reaction of 5-(4-aminophenyl)-hydantoin, 5-(4-aminobenzyl)-hydantoin and 5-(4-aminobenzyl)-thiohydantoin with the freshly prepared acyl isothiocyanates in moderate to excellent yields.

The data in Table 1 showed that some of these compounds, such as 71, 80 and $\mathbf{8 p}$ exhibit 91\%, 94\% and $87 \%$ inhibition rates against B. campestris, respectively, while they only have less than $25 \%$ inhibition rates against E. crus-galli at the concentration of $100 \mu \mathrm{g} / \mathrm{mL}$. Further tests in a greenhouse were performed and the results in Table 2 showed that $\mathbf{7 1 , 8 0}$ and $\mathbf{8 p}$ inhibit the growth of B. campestris with $100 \%, 100 \%$ and $95 \%$ efficacy after the post-emergence treatments, and only $23 \%$, $46 \%$ and $31 \%$ efficacy after the pre-emergence treatments at the dosage of $1000 \mathrm{~g} / \mathrm{ha}$. However they exhibited less than $15 \%$ efficacy against E. crus-galli after the post-emergence or pre-emergence treatments. These results indicated that these compounds exhibit certain selectively herbicidal activities.

The data in Table 3 showed that only $\mathbf{7 a}, \mathbf{7 b}, \mathbf{7 c}, \mathbf{7 d}$ and $\mathbf{7 h}$ exhibit more than $70 \%$ inhibition rates against Fusarium oxysporum, and the others including 8 and 9 (data not shown in Table 3) have less than 55\% inhibition rates against Alternaria solani, Botryospuaeria berengeriana, Cercospora arachidcola and Fusahum graminearum at the concentration of $100 \mu \mathrm{g} / \mathrm{mL}$. The inhibition rates of $\mathbf{7 a}, \mathbf{7 b}, \mathbf{7 c}, \mathbf{7 d}$ and $\mathbf{7 h}$ against $F$. oxysporum were $74 \%, 79 \%, 79 \%, 71 \%$ and $71 \%$, respectively. They are weaker when compared to carbendazin (the positive control) against $F$. oxysporum and need further structure modification to increase the fungicidal activity.

Table 1. The herbicidal activities (inhibition rate, \%) of compounds 7, 8 and 9.

\begin{tabular}{ccccccccc}
\hline Compd. & B. campestris & E. crus-galli & Compd. & B. campestris & E. crus-galli & Compd. & B. campestris & E. crus-galli \\
\hline $7 \mathrm{a}$ & 20 & 10 & $8 \mathrm{a}$ & 2 & 15 & $9 \mathrm{a}$ & 3 & 10 \\
$7 \mathrm{~b}$ & 38 & 0 & $8 \mathrm{~b}$ & 12 & 15 & $9 \mathrm{~b}$ & 44 & 10 \\
$7 \mathrm{c}$ & 0 & 15 & $8 \mathrm{c}$ & 16 & 20 & $9 \mathrm{c}$ & 40 & 5 \\
$7 \mathrm{~d}$ & 39 & 0 & $8 \mathrm{~d}$ & 14 & 5 & $9 \mathrm{~d}$ & 33 & 15 \\
$7 \mathrm{e}$ & 29 & 10 & $8 \mathrm{e}$ & 0 & 5 & $9 \mathrm{e}$ & 0 & 15 \\
$7 \mathrm{f}$ & 0 & 5 & $8 \mathrm{f}$ & 25 & 0 & $9 \mathrm{f}$ & 0 & 10 \\
$7 \mathrm{~g}$ & 49 & 10 & $8 \mathrm{~g}$ & 4 & 10 & $9 \mathrm{~g}$ & 6 & 25 \\
$7 \mathrm{~h}$ & 23 & 15 & $8 \mathrm{~h}$ & 10 & 10 & $9 \mathrm{~h}$ & 29 & 25 \\
$7 \mathrm{i}$ & 0 & 0 & $8 \mathrm{i}$ & 11 & 15 & $9 \mathrm{i}$ & 18 & 25 \\
$7 \mathrm{j}$ & 14 & 5 & $8 \mathrm{j}$ & 25 & 0 & $9 \mathrm{j}$ & 6 & 10 \\
$7 \mathrm{k}$ & 6 & 20 & $8 \mathrm{k}$ & 0 & 10 & $9 \mathrm{k}$ & 0 & 5 \\
71 & 91 & 0 & 81 & 0 & 0 & 91 & 0 & 10 \\
& & & $8 \mathrm{~m}$ & 0 & 0 & $9 \mathrm{~m}$ & 33 & 5 \\
& & & $8 \mathrm{n}$ & 7 & 10 & & & \\
& & & $8 \mathrm{o}$ & 94 & 10 & & & \\
\hline
\end{tabular}


Table 2. The herbicidal activities (efficacy, \%) of compounds $7 \mathbf{l}, \mathbf{8 0}$ and $\mathbf{8 p}$ in greenhouse test.

\begin{tabular}{ccccc}
\hline \multirow{2}{*}{ Compd. } & \multicolumn{2}{c}{ B. campestris } & \multicolumn{2}{c}{ E. crus-galli } \\
& Pre-emergence & Post-emergence & Pre-emergence & Post-emergence \\
\hline 71 & 23 & 100 & 0 & 0 \\
$\mathbf{8 0}$ & 46 & 100 & 0 & 10 \\
$\mathbf{8 p}$ & 31 & 95 & 10 & 15 \\
\hline
\end{tabular}

Table 3. The fungicidal activities (inhibition rate, \%) of compounds 7 against several plant fungi.

\begin{tabular}{cccccc}
\hline Compd. & F. oxysporum & A. Solani & B. berengeriana & C. arachidcola & F. graminearum \\
\hline $\mathbf{7 a}$ & 74 & 20 & 32 & 0 & 25 \\
$7 \mathbf{b}$ & 79 & 24 & 23 & 0 & 54 \\
$7 \mathbf{c}$ & 79 & 24 & 23 & 0 & 40 \\
$\mathbf{7 d}$ & 71 & 21 & 7 & 6 & 44 \\
$\mathbf{7 e}$ & 59 & 17 & 30 & 6 & 25 \\
$\mathbf{7 f}$ & 24 & 27 & 28 & 6 & 10 \\
$7 \mathbf{g}$ & 24 & 27 & 37 & 6 & 18 \\
$7 \mathbf{h}$ & 71 & 3 & 20 & 0 & 40 \\
$7 \mathbf{i}$ & 24 & 13 & 25 & 6 & 16 \\
$7 \mathbf{j}$ & 24 & 27 & 37 & 6 & 18 \\
$7 \mathbf{k}$ & 41 & 20 & 23 & 0 & 40 \\
$\mathbf{7 1}$ & 15 & 17 & 40 & 0 & 12 \\
Carbendazin & 100 & 44 & 97 & 8 & 100 \\
\hline
\end{tabular}

\section{Experimental Section}

\subsection{General Information}

All reactions were performed under room temperature with magnetic stirring. Unless otherwise stated, all reagents were purchased from commercial suppliers and used without further purification. Organic solutions were concentrated under reduced pressure using a rotary evaporator or oil pump. Melting points were measured on a Yanagimoto apparatus (Yanagimoto MFG Co., Kyoto, Japan) and uncorrected. Infrared spectra were recorded using a Shimadzu IR-435 instrument with KBr plates. ${ }^{1} \mathrm{H}$ NMR spectra were obtained on Bruker DPX 300 spectrometer (Bruker Biospin Co., Stuttgart, Germany) with DMSO- $d_{6}$ as a solvent and TMS as an internal standard. Elemental analysis was performed on a Vario EL instrument (Elementar Vario Micro Cube, Hanau, Germany).

\subsection{Synthesis}

3.2.1. Synthesis of 5-(4-Aminobenzyl)-, 5-(4-Aminophenyl)-Hydantoin (4 and 5), 5-(4-Aminobenzyl)-Thiohydantoin (6) and Acyl Isothiocyanate Derivatives

The synthesis of the intermediates 4, 5 and $\mathbf{6}$ were carried out according to the protocols in our previous paper and their spectral data were identical with that in the reference [37]. To a suspension of 
benzoic acid derivative $(20 \mathrm{mmol})$ in $25 \mathrm{~mL}$ of $\mathrm{CH}_{2} \mathrm{Cl}_{2}$ in a $50 \mathrm{~mL}$ three-necked flask, $8 \mathrm{~mL} \mathrm{SOCl}_{2}$ and a drop of $\mathrm{N}, \mathrm{N}$-dimethylformamide (DMF) were added. After stirring at room temperature for $3 \mathrm{~h}$, the solution was evaporated. The resulting acyl chloride was dissolved in $15 \mathrm{~mL}$ of anhydrous acetonitrile and added to a solution of $20 \mathrm{mmol}$ potassium thiocyanate in $25 \mathrm{~mL}$ of acetonitrile with two drops of polyethylene glycol-400 (PEG-400). After stirring at room temperature for $2 \mathrm{~h}$, the mixture was filtered to give the acyl isothiocyanate derivatives, which were used without further purification $[14,15]$.

\subsubsection{General Procedure for the Synthesis of Compounds 7, 8 and 9}

To a stirred solution of $20 \mathrm{mmol} 5$-(4-aminophenyl)-hydantoin (4), or 5-(4-aminobenzyl)-hydantoin (5) or 5-(4-aminobenzyl)-2-thiohydantoin (6) in $20 \mathrm{~mL}$ of anhydrous acetonitrile, the acyl isothiocyanate solution in acetonitrile freshly prepared were added dropwise at ambient temperature. The reaction was monitored by TLC. After leaving it overnight, the reaction was stopped and the product was filtered. The products were further purified by recrystallization using DMF-EtOH- $\mathrm{H}_{2} \mathrm{O}$ to afford the compounds 7,8 and 9.

$N$-((4-(2,4-dioxoimidazolidin-5-yl)phenyl)carbamothioyl)benzamide 7a, white solid, yield 82\%, m.p. $140-142{ }^{\circ} \mathrm{C},{ }^{1} \mathrm{H}$ NMR (DMSO-d $\left.6,300 \mathrm{MHz}\right) \delta: 12.60$ (s, 1H, NH), 11.58 (s, 1H, NH), 10.82 (s, 1H, NH), 8.43 (s, 1H, NH), 7.97 (d, J=8.4 Hz, 2H, ArH), 7.73-7.52 (m, 5H, ArH), 7.38 (d, $J=8.4 \mathrm{~Hz}, 2 \mathrm{H}, \mathrm{ArH}), 5.20$ (s, 1H, CH); IR (KBr) v: 3160, 3053, 1787, 1725, 1672, $1597 \mathrm{~cm}^{-1}$. Anal calcd. for $\mathrm{C}_{17} \mathrm{H}_{14} \mathrm{~N}_{4} \mathrm{O}_{3} \mathrm{~S}$ : C 57.62, H 3.98, N 15.81; Found: C 57.59, H 3.90, N 15.78.

$N$-((4-(2,4-dioxoimidazolidin-5-yl)phenyl)carbamothioyl)-4-fluorobenzamide 7b, white solid, yield 70\%, m.p. ${ }^{146-148}{ }^{\circ} \mathrm{C},{ }^{1} \mathrm{H}$ NMR (DMSO-d $\left.6,300 \mathrm{MHz}\right) \delta: 12.55$ (s, 1H, NH), 11.64 (s, 1H, NH), $10.83(\mathrm{~s}, 1 \mathrm{H}, \mathrm{NH}), 8.44(\mathrm{~s}, 1 \mathrm{H}, \mathrm{NH}), 8.09-8.03(\mathrm{~m}, 2 \mathrm{H}, \mathrm{ArH}), 7.70(\mathrm{~d}, 2 \mathrm{H}, J=8.4 \mathrm{~Hz}, \mathrm{ArH})$, 7.42-7.32 (m, 4H, ArH), 5.20 (s, 1H, CH); IR (KBr) v: 3125, 3041, 1784, 1729, 1666, $1601 \mathrm{~cm}^{-1}$. Anal calcd. for $\mathrm{C}_{17} \mathrm{H}_{13} \mathrm{FN}_{4} \mathrm{O}_{3} \mathrm{~S}$ : C 54.83, H 3.52, N 15.05; Found: C 54.80, H 3.50, N 15.01.

$\mathrm{N}$-((4-(2,4-dioxoimidazolidin-5-yl)phenyl)carbamothioyl)-4-chlorobenzamide 7c, white solid, yield 55\%, m.p.230-232 ${ }^{\circ} \mathrm{C},{ }^{1} \mathrm{H}$ NMR (DMSO-d $\left.6,300 \mathrm{MHz}\right) \delta: 12.50$ (s, 1H, NH), 11.69 (s, 1H, NH), 10.81 (s, 1H, NH), 8.43 (s, 1H, NH), 7.99 (d, $J=7.4 \mathrm{~Hz}, 2 \mathrm{H}, \mathrm{ArH}), 7.70$ (d, $J=8.4 \mathrm{~Hz}, 2 \mathrm{H}, \operatorname{ArH})$, 7.62 (d, $J=7.4 \mathrm{~Hz}, 2 \mathrm{H}, \mathrm{ArH}), 7.37$ (d, $J=8.4 \mathrm{~Hz}, 2 \mathrm{H}, \mathrm{ArH}), 5.20$ (s, 1H, CH); IR (KBr) v: 3145, $3035,1783,1728,1670,1593 \mathrm{~cm}^{-1}$; Anal calcd. for $\mathrm{C}_{17} \mathrm{H}_{13} \mathrm{ClN}_{4} \mathrm{O}_{3} \mathrm{~S}$ : C 52.51, H 3.37, N 14.41; Found: C 52.53, H 3.41, N 14.36.

$N$-((4-(2,4-dioxoimidazolidin-5-yl)phenyl)carbamothioyl)-4-methylbenzamide $\mathbf{7 d ,}$ white solid, yield 53\%, m.p.220-222 ${ }^{\circ} \mathrm{C},{ }^{1} \mathrm{H}$ NMR (DMSO-d $\left.6,300 \mathrm{MHz}\right) \delta: 12.65$ (s, 1H, NH), 11.50 (s, 1H, NH), 10.82 (s, 1H, NH), 8.44 (s, 1H, NH), 7.91 (d, $J=7.5 \mathrm{~Hz}, 2 \mathrm{H}, \operatorname{ArH}), 7.71$ (d, $J=8.4 \mathrm{~Hz}, 2 \mathrm{H}, \operatorname{ArH})$, 7.39-7.34 (m, 4H, ArH), 5.20 (s, 1H, CH), 2.40 (s, 3H, $\left.\mathrm{CH}_{3}\right)$; IR (KBr) v: 3150, 3035, 1786, 1726, 1664, $1598 \mathrm{~cm}^{-1}$; Anal calcd. for $\mathrm{C}_{18} \mathrm{H}_{16} \mathrm{~N}_{4} \mathrm{O}_{3} \mathrm{~S}$ : C 58.68, H 4.38, N 15.21; Found: C 58.63, H 4.40, N 15.11.

$N$-((4-(2,4-dioxoimidazolidin-5-yl)phenyl)carbamothioyl)-2-methylbenzamide 7e, white solid, yield 54\%, m.p.138-140 ${ }^{\circ} \mathrm{C},{ }^{1} \mathrm{H}$ NMR (DMSO-d 6 , $\left.300 \mathrm{MHz}\right) \delta: 12.62$ (s, 1H, NH), 11.51 (s, 1H, NH), 10.82 (s, 1H, NH), 8.43 (s, 1H, NH), 7.84-7.70 (m, 4H, ArH), 7.47-7.37 (m, 4H, ArH), 5.20 (s, 1H, CH), 
$2.40\left(\mathrm{~s}, 3 \mathrm{H}, \mathrm{CH}_{3}\right)$; IR (KBr) v: 3170, 3045, 1789, 1715, 1677, $1598 \mathrm{~cm}^{-1}$; Anal calcd. for $\mathrm{C}_{18} \mathrm{H}_{16} \mathrm{~N}_{4} \mathrm{O}_{3} \mathrm{~S}$ : C 58.68, H 4.38, N 15.21; Found: C 58.62, H 4.55, N 15.35.

$N$-((4-(2,4-dioxoimidazolidin-5-yl)phenyl)carbamothioyl)-2-chlorobenzamide 7f, white solid, yield 55\%, m.p.222-224 ${ }^{\circ} \mathrm{C},{ }^{1} \mathrm{H}$ NMR (DMSO-d 6 , $300 \mathrm{MHz}$ ) $\delta: 12.34$ (s, 1H, NH), 12.03 (s, 1H, NH), 10.83 (s, 1H, NH), 8.44 (s, 1H, NH), 7.72 (d, $J=7.4 \mathrm{~Hz}, 2 \mathrm{H}, \operatorname{ArH}), 7.65-7.43$ (m, 4H, ArH), 7.38 (d, $J=7.4 \mathrm{~Hz}, 2 \mathrm{H}, \mathrm{ArH}), 5.21$ (s, 1H, CH); IR (KBr) v: 3301, 3056, 1783, 1715, 1694, $1593 \mathrm{~cm}^{-1}$; Anal calcd. for $\mathrm{C}_{17} \mathrm{H}_{13} \mathrm{ClN}_{4} \mathrm{O}_{3} \mathrm{~S}$ : C 52.51, H 3.37, N 14.41; Found: C 52.60, H 3.41, N 14.44.

$N$-((4-(2,4-dioxoimidazolidin-5-yl)phenyl)carbamothioyl)-2-nitrobenzamide 7g, white solid, yield 63\%, m.p.234-236 ${ }^{\circ} \mathrm{C},{ }^{1} \mathrm{H}$ NMR (DMSO-d 6 , $300 \mathrm{MHz}$ ) $\delta: 12.25$ (s, 1H, NH), 12.17 (s, 1H, NH), 10.84 (s, 1H, NH), $8.46(\mathrm{~s}, 1 \mathrm{H}, \mathrm{NH}), 8.23$ (d, $J=8.1 \mathrm{~Hz}, 1 \mathrm{H}, \mathrm{ArH}), 7.94-7.71(\mathrm{~m}, 5 \mathrm{H}, \mathrm{ArH}), 7.39$ (d, $J=7.4 \mathrm{~Hz}, 2 \mathrm{H}, \mathrm{ArH}), 5.22$ (s, 1H, CH); IR (KBr) v: 3146, 3034, 1764, 1716, 1690, $1593 \mathrm{~cm}^{-1}$; Anal calcd. for $\mathrm{C}_{17} \mathrm{H}_{13} \mathrm{~N}_{5} \mathrm{O}_{5} \mathrm{~S}$ : C 51.12, H 3.28, N 17.54; Found: C 51.14, H 3.21, N 17.25.

$N$-((4-(2,4-dioxoimidazolidin-5-yl)phenyl)carbamothioyl)-3-methylbenzamide $\mathbf{7 h}$, white solid, yield 54\%, m.p. $158-160{ }^{\circ} \mathrm{C},{ }^{1} \mathrm{H}$ NMR (DMSO-d $\left.6,300 \mathrm{MHz}\right) \delta: 12.62$ (s, 1H, NH), 11.54 (s, 1H, NH), 10.83 (s, 1H, NH), 8.45 (s, 1H, NH), 7.83-7.70 (m, 4H, ArH), 7.49-7.36 (m, 4H, ArH), $5.21(\mathrm{~s}, 1 \mathrm{H}, \mathrm{CH})$, $2.39\left(\mathrm{~s}, 3 \mathrm{H}, \mathrm{CH}_{3}\right)$; IR $(\mathrm{KBr})$ v: 3210, 3047, 1769, 1726, 1668, $1600 \mathrm{~cm}^{-1}$; Anal calcd. for $\mathrm{C}_{18} \mathrm{H}_{16} \mathrm{~N}_{4} \mathrm{O}_{3} \mathrm{~S}$ : C 58.68, H 4.38, N 15.21; Found: C 58.64, H 4.40, N 15.16.

$N$-((4-(2,4-dioxoimidazolidin-5-yl)phenyl)carbamothioyl)-3-nitrobenzamide 7i, white solid, yield 52\%, m.p.162-164 ${ }^{\circ} \mathrm{C},{ }^{1} \mathrm{H}$ NMR (DMSO-d $\left.6,300 \mathrm{MHz}\right) \delta: 12.42$ (s, 1H, NH), 12.04 (s, 1H, NH), 10.83 (s, 1H, NH), $8.78(\mathrm{~d}, J=2.1 \mathrm{~Hz}, 1 \mathrm{H}, \operatorname{ArH}), 8.51-8.47$ (m, 1H, ArH), 8.45 (s, 1H, NH), 8.37 (dd, $J=7.8,2.4 \mathrm{~Hz}, 1 \mathrm{H}, \mathrm{ArH}), 7.84(\mathrm{t}, J=7.8 \mathrm{~Hz}, 1 \mathrm{H}, \mathrm{ArH}), 7.72$ (d, $J=8.4 \mathrm{~Hz}, 2 \mathrm{H}, \mathrm{ArH}), 7.39$ (d, $J=8.4 \mathrm{~Hz}, 2 \mathrm{H}, \mathrm{ArH}), 5.21(\mathrm{~s}, 1 \mathrm{H}, \mathrm{CH})$; IR (KBr) v: 3229, 3047, 1776, 1723, 1677, $1600 \mathrm{~cm}^{-1}$; Anal calcd. for $\mathrm{C}_{17} \mathrm{H}_{13} \mathrm{~N}_{5} \mathrm{O}_{5} \mathrm{~S}$ : C 51.12, H 3.28, N 17.54; Found: C 51.02, H 3.30, N 17.54.

$N$-((4-(2,4-dioxoimidazolidin-5-yl)phenyl)carbamothioyl)thiophene-2-carboxamide 7j, white solid, yield 80\%, m.p. $226-228{ }^{\circ} \mathrm{C},{ }^{1} \mathrm{H}$ NMR (DMSO-d $\left.6,300 \mathrm{MHz}\right) \delta: 12.44$ (s, 1H, NH), 11.64 (s, 1H, NH), $10.81(\mathrm{~s}, 1 \mathrm{H}, \mathrm{NH}), 8.43$ (s, 1H, NH), 8.38 (d, J=3.8 Hz, 1H, ThH), 8.05 (d, $J=5.0 \mathrm{~Hz}, 1 \mathrm{H}, \mathrm{ThH})$, $7.70(\mathrm{~d}, J=7.4 \mathrm{~Hz}, 2 \mathrm{H}, \mathrm{ArH}), 7.36$ (d, $J=7.4 \mathrm{~Hz}, 2 \mathrm{H}, \mathrm{ArH}), 7.26$ (dd, $J=3.8,5.0 \mathrm{~Hz}, 1 \mathrm{H}, \mathrm{ThH}), 5.20$ (s, 1H, CH); IR (KBr) v: 3138, 3043, 1778, 1722, 1657, $1592 \mathrm{~cm}^{-1}$. Anal calcd. for $\mathrm{C}_{15} \mathrm{H}_{12} \mathrm{~N}_{4} \mathrm{O}_{3} \mathrm{~S}_{2}: \mathrm{C}$ 49.99, H 3.36, N 15.55; Found: C 49.97, H 3.39, N 15.56.

2-Chloro- $N$-((4-(2,4-dioxoimidazolidin-5-yl)phenyl)carbamothioyl)isonicotinamide 7k, white solid, yield 73\%, m.p. $212-214{ }^{\circ} \mathrm{C},{ }^{1} \mathrm{H}$ NMR (DMSO-d $\left.6,300 \mathrm{MHz}\right) \delta: 12.25$ (s, 1H, NH), 11.96 (s, 1H, NH), 10.81 (s, 1H, NH), 8.62 (d, $J=5.4 \mathrm{~Hz}, 1 \mathrm{H}, \mathrm{PyH}), 8.43$ (s, 1H, NH), 7.99 (s, 1H, PyH), 7.84 (dd, $J=1.2,5.4 \mathrm{~Hz}, 1 \mathrm{H}, \mathrm{PyH}), 7.70$ (d, $J=7.4 \mathrm{~Hz}, 2 \mathrm{H}, \mathrm{ArH}), 7.39$ (d, $J=7.4 \mathrm{~Hz}, 2 \mathrm{H}, \mathrm{ArH}), 5.20$ (s, 1H, $\mathrm{CH}$ ); IR (KBr) v: 3150, 3042, 1765, 1719, 1666, $1593 \mathrm{~cm}^{-1}$. Anal calcd. for $\mathrm{C}_{16} \mathrm{H}_{12} \mathrm{ClN}_{5} \mathrm{O}_{3} \mathrm{~S}: \mathrm{C} 49.30$, H 3.10, N 17.97; Found: C 49.26, H 3.08, N 17.90.

2-(2,4-dichlorophenoxy)- $N$-((4-(2,4-dioxoimidazolidin-5-yl)phenyl)carbamothioyl)acetamide 7l, white solid, yield 59\%, m.p. $258-260{ }^{\circ} \mathrm{C},{ }^{1} \mathrm{H}$ NMR (DMSO-d $\left.6,300 \mathrm{MHz}\right) \delta: 11.85$ (s, 1H, NH), 10.77 (s, 1H, NH), 10.24 (s, 1H, NH), 8.37 (s, 1H, NH), 7.63-7.60 (m, 3H, ArH), 7.37 (dd, J=2.4, 8.4 Hz, 1H, ArH), 7.27 (d, J=7.5 Hz, 2H, ArH), 7.09 (d, J=8.4 Hz, 1H, ArH), 5.11 (s, 1H, CH), 4.86 (s, 2H, $\mathrm{CH}_{2}$ ); IR (KBr) v: 3288, 3060, 1780, 1740, 1682, $1600 \mathrm{~cm}^{-1}$. Anal calcd. for $\mathrm{C}_{18} \mathrm{H}_{14} \mathrm{Cl}_{2} \mathrm{~N}_{4} \mathrm{O}_{4} \mathrm{~S}$ : C 47.69, H 3.11, N 12.36; Found: C 47.58, H 3.12, N 12.34. 
$N$-((4-((2,4-dioxoimidazolidin-5-yl)methyl)phenyl)carbamothioyl)benzamide 8a, white solid, yield 93\%, m.p. $240-242{ }^{\circ} \mathrm{C},{ }^{1} \mathrm{H}$ NMR (DMSO-d $\left.6,300 \mathrm{MHz}\right) \delta: 12.61$ (s, 1H, NH), 11.53 (s, 1H, NH), $10.48(\mathrm{~s}, 1 \mathrm{H}, \mathrm{NH}), 7.99-7.96(\mathrm{~m}, 3 \mathrm{H}, \mathrm{ArH}+\mathrm{NH}), 7.70-7.64(\mathrm{~m}, 3 \mathrm{H}, \mathrm{ArH}), 7.57-7.52(\mathrm{~m}, 2 \mathrm{H}$, $\operatorname{ArH}), 7.23(\mathrm{~d}, J=7.8 \mathrm{~Hz}, 2 \mathrm{H}, \mathrm{ArH}), 4.37-4.34(\mathrm{~m}, 1 \mathrm{H}, \mathrm{CH}), 2.99-2.89\left(\mathrm{~m}, 2 \mathrm{H}, \mathrm{CH}_{2}\right)$; IR $(\mathrm{KBr})$ v: $3172,3064,1766,1726,1668,1597 \mathrm{~cm}^{-1}$. Anal calcd. for $\mathrm{C}_{18} \mathrm{H}_{16} \mathrm{~N}_{4} \mathrm{O}_{3} \mathrm{~S}: \mathrm{C} 58.68, \mathrm{H} 4.38, \mathrm{~N} 15.21$; Found: C 58.98, H 4.30, N 15.25.

$N$-((4-((2,4-dioxoimidazolidin-5-yl)methyl)phenyl)carbamothioyl)-4-flurobenzamide $\quad \mathbf{8 b}, \quad$ white solid, yield 83\%, m.p. $216-218{ }^{\circ} \mathrm{C},{ }^{1} \mathrm{H}$ NMR (DMSO-d $\left.6,300 \mathrm{MHz}\right) \delta: 12.56$ (s, 1H, NH), 11.58 (s, 1H, $\mathrm{NH}), 10.48(\mathrm{~s}, 1 \mathrm{H}, \mathrm{NH}), 8.09-8.03(\mathrm{~m}, 2 \mathrm{H}, \mathrm{ArH}), 7.96(\mathrm{~s}, 1 \mathrm{H}, \mathrm{NH}), 7.64(\mathrm{~d}, J=8.0 \mathrm{~Hz}, 2 \mathrm{H}, \operatorname{ArH})$, 7.40-7.35 (m, 2H, ArH), 7.23 (d, $J=8.0 \mathrm{~Hz}, 2 \mathrm{H}, \mathrm{ArH}), 4.37-4.34$ (m, 1H, CH,), 2.97-2.94 (m, 2H, $\mathrm{CH}_{2}$ ); IR (KBr) v: $3178,3060,1762,1727,1669,1600 \mathrm{~cm}^{-1}$. Anal calcd for $\mathrm{C}_{18} \mathrm{H}_{15} \mathrm{FN}_{4} \mathrm{O}_{3} \mathrm{~S}: \mathrm{C} 55.95$, H 3.91, N 14.50; Found: C 55.86, H 4.01, N 14.51.

$N$-((4-((2,4-dioxoimidazolidin-5-yl)methyl)phenyl)carbamothioyl)-4-chlorobenzamide $\mathbf{8 c}$, white solid, yield 63\%, m.p. $232-234{ }^{\circ} \mathrm{C},{ }^{1} \mathrm{H}$ NMR (DMSO-d $\left.6,300 \mathrm{MHz}\right) \delta: 12.51$ (s, 1H, NH), 11.64 (s, 1H, $\mathrm{NH}), 10.49$ (s, 1H, NH), 7.98-7.93 (m, 3H, ArH + NH), 7.66-7.56 (m, 4H, ArH), 7.23 (d, 2H, $J=8.4 \mathrm{~Hz}, \mathrm{ArH}), 4.37-4.33(\mathrm{~m}, 1 \mathrm{H}, \mathrm{CH}), 2.95-2.93$ (m, 2H, $\left.\mathrm{CH}_{2}\right)$; IR (KBr) v: 3155, 3034, 1758, 1726, 1666, $1594 \mathrm{~cm}^{-1}$. Anal calcd. for $\mathrm{C}_{18} \mathrm{H}_{15} \mathrm{ClN}_{4} \mathrm{O}_{3} \mathrm{~S}$ : C 53.67, $\mathrm{H} 3.75, \mathrm{~N} 13.91$; Found: $\mathrm{C} 53.78$, H 3.79, N 13.90.

$N$-((4-((2,4-dioxoimidazolidin-5-yl)methyl)phenyl)carbamothioyl)-4-methylbenzamide 8d, white solid, yield 66\%, m.p. $228-230{ }^{\circ} \mathrm{C},{ }^{1} \mathrm{H}$ NMR (DMSO-d $\left.6,300 \mathrm{MHz}\right) \delta: 12.67$ (s, 1H, NH), 11.43 (s, 1H, $\mathrm{NH}), 10.48$ (s, 1H, NH), $7.96(\mathrm{~s}, 1 \mathrm{H}, \mathrm{NH}), 7.91$ (d, $J=8.1 \mathrm{~Hz}, 2 \mathrm{H}, \mathrm{ArH}), 7.65$ (d, $J=8.4 \mathrm{~Hz}, 2 \mathrm{H}$, $\operatorname{ArH}), 7.35$ (d, $J=8.1 \mathrm{~Hz}, 2 \mathrm{H}, \mathrm{ArH}), 7.23$ (d, $J=8.4 \mathrm{~Hz}, 2 \mathrm{H}, \mathrm{ArH}), 4.37-4.34$ (m, 1H, CH), 2.96-2.93 $\left(\mathrm{m}, 2 \mathrm{H}, \mathrm{CH}_{2}\right), 2.37$ (s, 3H, $\left.\mathrm{CH}_{3}\right)$; IR (KBr) v: 3171, 3067, 1767, 1730, 1668, $1598 \mathrm{~cm}^{-1}$. Anal calcd. for $\mathrm{C}_{19} \mathrm{H}_{18} \mathrm{~N}_{4} \mathrm{O}_{3} \mathrm{~S}$ : C 59.67, H 4.74, N 14.65; Found: C 59.58, H 4.67, N 14.48.

$N$-((4-((2,4-dioxoimidazolidin-5-yl)methyl)phenyl)carbamothioyl)-4-methoxybenzamide 8e, white solid, yield 69\%, m.p. $224-226{ }^{\circ} \mathrm{C},{ }^{1} \mathrm{H}$ NMR (DMSO-d $\left.6,300 \mathrm{MHz}\right) \delta: 12.74$ (s, 1H, NH), 11.39 (s, 1H, NH), 10.50 (s, 1H, NH), 8.02 (d, $J=8.7 \mathrm{~Hz}, 2 \mathrm{H}, \mathrm{ArH}$ ), 7.99 (s, 1H, NH), 7.64 (d, $J=8.4 \mathrm{~Hz}, 2 \mathrm{H}$, $\operatorname{ArH}), 7.22$ (d, $J=8.4 \mathrm{~Hz}, 2 \mathrm{H}, \operatorname{ArH}), 7.07$ (d, $J=8.7 \mathrm{~Hz}, 2 \mathrm{H}, \mathrm{ArH}), 4.37-4.34$ (m, 1H, CH), 3.86 (s, $\left.3 \mathrm{H}, \mathrm{OCH}_{3}\right), 2.95-2.93\left(\mathrm{~m}, 2 \mathrm{H}, \mathrm{CH}_{2}\right)$; IR $(\mathrm{KBr})$ v: 3176, 3051, 1766, 1726, 1667, $1596 \mathrm{~cm}^{-1}$. Anal calcd. for $\mathrm{C}_{19} \mathrm{H}_{18} \mathrm{~N}_{4} \mathrm{O}_{4} \mathrm{~S}$ : C 57.27, H 4.55, N 14.16; Found: C 57.22, H 4.55, N 14.03.

$N$-((4-((2,4-dioxoimidazolidin-5-yl)methyl)phenyl)carbamothioyl)-4-nitrobenzamide $\quad \mathbf{8 f}, \quad$ yellow solid, yield 82\%, m.p. $226-228{ }^{\circ} \mathrm{C},{ }^{1} \mathrm{H}$ NMR (DMSO-d $\left.6,300 \mathrm{MHz}\right) \delta: 12.75$ (s, 1H, NH), 11.47(s, 1H, $\mathrm{NH}), 10.44(\mathrm{~s}, 1 \mathrm{H}, \mathrm{NH}), 8.38-7.93(\mathrm{~m}, 5 \mathrm{H}, \mathrm{ArH}+\mathrm{NH}), 7.35-7.20(\mathrm{~m}, 4 \mathrm{H}, \mathrm{ArH}), 4.38-4.33(\mathrm{~m}, 1 \mathrm{H}$, $\mathrm{CH}), 2.97-2.92\left(\mathrm{~m}, 2 \mathrm{H}, \mathrm{CH}_{2}\right)$; IR (KBr) v: 3112, 3047, 1768, 1728, 1667, $1592 \mathrm{~cm}^{-1}$. Anal calcd. for $\mathrm{C}_{18} \mathrm{H}_{15} \mathrm{~N}_{5} \mathrm{O}_{5} \mathrm{~S}$ : C 52.30, H 3.66, N 16.94; Found: C 52.23, H 3.68, N 16.85.

$N$-((4-((2,4-dioxoimidazolidin-5-yl)methyl)phenyl)carbamothioyl)-2-methylbenzamide $\mathbf{8 g}$, white solid, yield 56\%, m.p. $212-214{ }^{\circ} \mathrm{C},{ }^{1} \mathrm{H}$ NMR (DMSO-d $\left.6,300 \mathrm{MHz}\right) \delta: 12.56$ (s, 1H, NH), 11.68 (s, 1H, $\mathrm{NH}), 10.48$ (s, 1H, NH), 7.96 (s, 1H, NH), 7.67 (d, J=8.4 Hz, 2H, ArH), 7.51 7.22 (m, 6H, ArH), 4.37-4.34 (m, 1H, CH), 2.96-2.91 (m, 2H, $\left.\mathrm{CH}_{2}\right), 2.42$ (s, 3H, $\left.\mathrm{CH}_{3}\right)$; IR (KBr) v: 3181, 3066, 1767, 1722, 1673, $1595 \mathrm{~cm}^{-1}$. Anal calcd. for $\mathrm{C}_{19} \mathrm{H}_{18} \mathrm{~N}_{4} \mathrm{O}_{3} \mathrm{~S}$ : C 59.67, H 4.74, N 14.65; Found: C 59.69, H 4.75, N 14.74. 
$\mathrm{N}$-((4-((2,4-dioxoimidazolidin-5-yl)methyl)phenyl)carbamothioyl)-2-chlorobenzamide $\mathbf{8 h}$, white solid, yield 60\%, m.p. $216-218{ }^{\circ} \mathrm{C},{ }^{1} \mathrm{H}$ NMR (DMSO-d $\left.6,300 \mathrm{MHz}\right) \delta: 12.36$ (s, 1H, NH), 11.98 (s, 1H, $\mathrm{NH}), 10.49$ (s, 1H, NH), 7.97 (s, 1H, NH), 7.67-7.43 (m, 6H, ArH), 7.23 (d, J=8.5 Hz, 2H, ArH), 4.37-4.34 (m, 1H, CH), 2.99-2.93 (m, 2H, $\left.\mathrm{CH}_{2}\right)$; IR (KBr) v: 3194, 3060, 1766, 1717, 1679, 1592, $1537 \mathrm{~cm}^{-1}$. Anal calcd. for $\mathrm{C}_{18} \mathrm{H}_{15} \mathrm{ClN}_{4} \mathrm{O}_{3} \mathrm{~S}$ : C 53.67, H 3.75, N 13.91; Found: C 53.60, H 3.75, N 13.86.

$N$-((4-((2,4-dioxoimidazolidin-5-yl)methyl)phenyl)carbamothioyl)-2-methoxybenzamide 8i, white solid, yield 60\%, m.p. $216-218{ }^{\circ} \mathrm{C},{ }^{1} \mathrm{H}$ NMR (DMSO-d $\left.6,300 \mathrm{MHz}\right) \delta: 12.57$ (s 1H, NH), 12.21 (s, 1H, $\mathrm{NH}), 10.49$ (s, 1H, NH), $7.97(\mathrm{~s}, 1 \mathrm{H}, \mathrm{NH}), 7.94-7.91$ (m, 1H, ArH), 7.70-7.64 (m, 3H, ArH), 7.31-7.14 (m, 4H, ArH), 4.37-4.34 (m, 1H, CH), $4.01\left(\mathrm{~s}, 3 \mathrm{H}, \mathrm{OCH}_{3}\right), 2.96-2.94\left(\mathrm{~m}, 2 \mathrm{H}, \mathrm{CH}_{2}\right)$; IR (KBr) v: 3219, 3036, 1769, 1716, 1665, $1595 \mathrm{~cm}^{-1}$. Anal calcd. for $\mathrm{C}_{19} \mathrm{H}_{18} \mathrm{~N}_{4} \mathrm{O}_{4} \mathrm{~S}: \mathrm{C}$ 57.27, $\mathrm{H} 4.55$, N 14.16; Found: C 57.51, H 4.53, N 14.14.

$N$-((4-((2,4-dioxoimidazolidin-5-yl)methyl)phenyl)carbamothioyl)-2-nitrobenzamide $\quad \mathbf{8 j}, \quad$ yellow solid, yield 50\%, m.p. $222-224{ }^{\circ} \mathrm{C},{ }^{1} \mathrm{H}$ NMR (DMSO-d $\left.6,300 \mathrm{MHz}\right) \delta: 12.27$ (s, 1H, NH), 12.12 (s, 1H, $\mathrm{NH}), 10.49(\mathrm{~s}, 1 \mathrm{H}, \mathrm{NH}), 8.24-8.21(\mathrm{~m}, 1 \mathrm{H}, \mathrm{ArH}), 7.96(\mathrm{~s}, 1 \mathrm{H}, \mathrm{NH}), 7.94-7.77$ (m, 3H, ArH), $7.66(\mathrm{~d}, 2 \mathrm{H}, J=8.4 \mathrm{~Hz}, \operatorname{ArH}), 7.24$ (d, 2H, $J=8.4 \mathrm{~Hz}, \operatorname{ArH}), 4.38-4.34(\mathrm{~m}, 1 \mathrm{H}, \mathrm{CH}), 2.97-2.95$ $\left(\mathrm{m}, 2 \mathrm{H}, \mathrm{CH}_{2}\right)$; IR (KBr) v: 3219, 3036, 1769, 1716, 1665, $1595 \mathrm{~cm}^{-1}$. Anal calcd. for $\mathrm{C}_{18} \mathrm{H}_{15} \mathrm{~N}_{5} \mathrm{O}_{5} \mathrm{~S}: \mathrm{C}$ 52.30, H 3.66, N 16.94; Found: C 52.25, H 3.76, N 16.84.

$N$-((4-((2,4-dioxoimidazolidin-5-yl)methyl)phenyl)carbamothioyl)-3-methylbenzamide $\mathbf{8 k}$, white solid, yield 64\%, m.p. $220-221{ }^{\circ} \mathrm{C},{ }^{1} \mathrm{H}$ NMR (DMSO-d 6 , $\left.300 \mathrm{MHz}\right) \delta: 12.64$ (s, 1H, NH), 11.48 (s, 1H, $\mathrm{NH}), 10.49$ (s, 1H, NH), 7.97 (s, 1H, NH), 7.83-7.63 (m, 4H, ArH), 7.49-7.40 (m, 2H, ArH), 7.23 (d, $J=8.4 \mathrm{~Hz}, 2 \mathrm{H}, \mathrm{ArH}), 4.37-4.34(\mathrm{~m}, 1 \mathrm{H}, \mathrm{CH}), 2.97-2.92\left(\mathrm{~m}, 2 \mathrm{H}, \mathrm{CH}_{2}\right), 2.36\left(\mathrm{~s}, 3 \mathrm{H}, \mathrm{CH}_{3}\right)$; IR $(\mathrm{KBr})$ v: $3169,3056,1767,1726,1668,1596 \mathrm{~cm}^{-1}$. Anal calcd. for $\mathrm{C}_{19} \mathrm{H}_{18} \mathrm{~N}_{4} \mathrm{O}_{3} \mathrm{~S}$ : C 59.67, H 4.74, N 14.65; Found: C 59.62, H 4.75, N 14.54.

$N$-((4-((2,4-dioxoimidazolidin-5-yl)methyl)phenyl)carbamothioyl)-3-nitrobenzamide $\quad \mathbf{8 1 ,} \quad$ little yellow solid, yield 79\%, m.p. $168-170{ }^{\circ} \mathrm{C},{ }^{1} \mathrm{H}$ NMR (DMSO-d 6 , $300 \mathrm{MHz}$ ) $\delta: 12.43$ (s, 1H, NH), 12.01 (s, 1H, NH), 10.49 (s, 1H, NH), 8.78 (s, 1H, ArH), 8.51-8.36 (m, 2H, ArH), 7.99 (s, 1H, NH), 7.86-7.68 (m, 3H, ArH), 7.24 (d, $J=8.4 \mathrm{~Hz}, 2 \mathrm{H}, \mathrm{ArH}), 4.38-4.34$ (m, 1H, CH), 2.97-2.91 (m, 2H, $\mathrm{CH}_{2}$ ); IR (KBr) v: 3180, 3056, 1770, 1719, 1669, $1602 \mathrm{~cm}^{-1}$. Anal calcd. for $\mathrm{C}_{18} \mathrm{H}_{15} \mathrm{~N}_{5} \mathrm{O}_{5} \mathrm{~S}: \mathrm{C} 52.30, \mathrm{H}$ 3.66, N 16.94; Found: C 52.32, H 3.60, N 16.84.

$N$-((4-((2,4-dioxoimidazolidin-5-yl)methyl)phenyl)carbamothioyl)thiophene-2-carboxamide $\quad \mathbf{8 m}$, white solid, yield 82\%, m.p. $244-246{ }^{\circ} \mathrm{C},{ }^{1} \mathrm{H}$ NMR (DMSO-d $\left.6,300 \mathrm{MHz}\right) \delta: 12.46$ (s, 1H, NH), 11.58 (s, 1H, NH), $10.48(\mathrm{~s}, 1 \mathrm{H}, \mathrm{NH}), 8.39-8.37$ (m, 1H, ThH), 8.06-8.04 (m, 1H, ThH), $7.96(\mathrm{~s}, 1 \mathrm{H}, \mathrm{NH})$, $7.63(\mathrm{~d}, J=8.5 \mathrm{~Hz}, 2 \mathrm{H}, \mathrm{ArH}), 7.27-7.21(\mathrm{~m}, 3 \mathrm{H}, \mathrm{ArH}+\mathrm{ThH}), 4.37-4.32$ (m, 1H, CH), 2.96-2.91 (m, $2 \mathrm{H}, \mathrm{CH}_{2}$ ); IR (KBr) v: 3171, 3061, 1762, 1716, 1654, $1596 \mathrm{~cm}^{-1}$. Anal calcd. for $\mathrm{C}_{16} \mathrm{H}_{14} \mathrm{~N}_{4} \mathrm{O}_{3} \mathrm{~S}_{2}$ : C 51.32, H 3.77, N 14.96; Found: C 51.60, H 3.81, N 14.96.

2-Chloro- $N$-((4-((2,4-dioxoimidazolidin-5-yl)methyl)phenyl)carbamothioyl)isonicotinamide $\quad \mathbf{8 n}$, white solid, yield 80\%, m.p. 202-204 ${ }^{\circ} \mathrm{C},{ }^{1} \mathrm{H}$ NMR (DMSO-d $\left.6,300 \mathrm{MHz}\right) \delta$ : 12.25 (s, 1H, NH), 11.92 (s, 1H, NH), 10.48 (s, 1H, NH), 8.61 (d, J=5.1 Hz, 1H, PyH), 7.99-7.82 (m, 3H, PyH + NH), 7.63 (d, $J=8.3 \mathrm{~Hz}, 2 \mathrm{H}, \mathrm{ArH}), 7.24$ (d, $J=8.3 \mathrm{~Hz}, 2 \mathrm{H}, \mathrm{ArH}), 4.37-4.34(\mathrm{~m}, 1 \mathrm{H}, \mathrm{CH}), 2.99-2.89$ (m, 2H, $\mathrm{CH}_{2}$ ); IR (KBr) v: 3150, 3042, 1765, 1719, 1666, $1593 \mathrm{~cm}^{-1}$. Anal calcd. for $\mathrm{C}_{17} \mathrm{H}_{14} \mathrm{ClN}_{5} \mathrm{O}_{3} \mathrm{~S}: \mathrm{C} 50.56$, H 3.49, N 17.34; Found: C 50.70, H 3.57, N 17.27. 
2-(2,4-dichlorophenoxy)- $N$-((4-((2,4-dioxoimidazolidin-5-yl)methyl)phenyl)carbamothioyl)acetamide 8o, white solid, yield 71\%, m.p. 262-264 ${ }^{\circ} \mathrm{C},{ }^{1} \mathrm{H}$ NMR (DMSO-d $\left.6,300 \mathrm{MHz}\right) \delta: 11.95$ (s, 1H, $\mathrm{NH}), 10.41$ (s, 1H, NH), $10.14(\mathrm{~s}, 1 \mathrm{H}, \mathrm{NH}), 7.91$ (s, 1H, NH), 7.50 (d, J=8.5 Hz, 2H, ArH), 7.40 7.08 (m, 4H, ArH), 4.83 (s, 2H, CH 2$), 4.36-4.30$ (m, 1H, CH), 2.90-2.81 (m, 2H, $\left.\mathrm{CH}_{2}\right)$; IR (KBr) v: 3159, 3048, 1762, 1732, 1669, $1597 \mathrm{~cm}^{-1}$. Anal calcd. for $\mathrm{C}_{19} \mathrm{H}_{16} \mathrm{Cl}_{2} \mathrm{~N}_{4} \mathrm{O}_{4} \mathrm{~S}: \mathrm{C}$ 48.83, H 3.45, N 11.99; Found: C 48.72, H 3.55, N 11.92.

2-(4-dichlorophenoxy)- $N$-((4-((2,4-dioxoimidazolidin-5-yl)methyl)phenyl)carbamothioyl)acetamide 8p, white solid, yield 70\%, m.p. $210-212{ }^{\circ} \mathrm{C},{ }^{1} \mathrm{H}$ NMR (DMSO-d 6 , $\left.300 \mathrm{MHz}\right) \delta: 11.94$ (s, 1H, $\mathrm{NH}), 10.42(\mathrm{~s}, 1 \mathrm{H}, \mathrm{NH}), 10.06(\mathrm{~s}, 1 \mathrm{H}, \mathrm{NH}), 7.92(\mathrm{~s}, 1 \mathrm{H}, \mathrm{NH}), 7.52(\mathrm{~d}, J=8.5 \mathrm{~Hz}, 2 \mathrm{H}, \operatorname{ArH})$, 7.39 7.33 (m, 2H, ArH), 7.14 7.10 (m, 2H, ArH), 7.04 6.99 (m, 2H, ArH), $4.69\left(\mathrm{~s}, 2 \mathrm{H}, \mathrm{CH}_{2}\right)$, 4.34-4.28 (m, 1H, CH), 2.93-2.85 (m, 2H, $\left.\mathrm{CH}_{2}\right)$; IR (KBr) v: 3116, 3043, 1769, 1709, 1687, $1598 \mathrm{~cm}^{-1}$. Anal calcd. for $\mathrm{C}_{19} \mathrm{H}_{17} \mathrm{ClN}_{4} \mathrm{O}_{4} \mathrm{~S}$ : C 52.72, H 3.96, N 12.94; Found: C 52.62, H 3.86, N 12.90.

$N$-((4-((4-oxo-2-thioxoimidazolidin-5-yl)methyl)phenyl)carbamothioyl)benzamide 9a little yellow solid, yield 75\%, m.p. $240-242{ }^{\circ} \mathrm{C},{ }^{1} \mathrm{H}$ NMR (DMSO-d $\left.6,300 \mathrm{MHz}\right) \delta: 12.62$ (s, 1H, NH), 11.51 (s, 2H, NH), $10.09(\mathrm{~s}, 1 \mathrm{H}, \mathrm{NH}), 7.99 \sim 7.96(\mathrm{~m}, 2 \mathrm{H}, \mathrm{ArH}), 7.69 \sim 7.52(\mathrm{~m}, 5 \mathrm{H}, \mathrm{ArH}), 7.24 \sim 7.21(\mathrm{~m}, 2 \mathrm{H}$, ArH), 4.60-4.56 (m, 1H, CH), 3.01-2.96 (m, 2H, $\left.\mathrm{CH}_{2}\right)$; IR (KBr) v: 3185, 3081, 1772, 1742, 1655, $1597 \mathrm{~cm}^{-1}$. Anal calcd. for $\mathrm{C}_{18} \mathrm{H}_{16} \mathrm{~N}_{4} \mathrm{O}_{2} \mathrm{~S}_{2}$ : C 56.23, H 4.19, N 14.57; Found: C 56.16, H 4.21, N 14.55.

$N$-((4-((4-oxo-2-thioxoimidazolidin-5-yl)methyl)phenyl)carbamothioyl)-4-flurobenzamide 9b little yellow solid, yield 56\%, m.p. 238-240 ${ }^{\circ} \mathrm{C},{ }^{1} \mathrm{H}$ NMR (DMSO-d $6,300 \mathrm{MHz}$ ) $\delta: 12.57$ (s, 1H, NH), 11.59 (s, 1H, NH), $11.51(\mathrm{~s}, 1 \mathrm{H}, \mathrm{NH}), 10.10(\mathrm{~s}, 1 \mathrm{H}, \mathrm{NH}), 8.09 \sim 8.04(\mathrm{~m}, 2 \mathrm{H}, \mathrm{ArH}), 7.67 \sim 7.64(\mathrm{~m}, 2 \mathrm{H}$, ArH), 7.41 7.35 (m, 2H, ArH), 7.24 7.21 (m, 2H, ArH), 4.60-4.56 (m, 1H, CH), 3.01-2.95 (m, 2H, $\mathrm{CH}_{2}$ ); IR (KBr) v: 3309, 3047, 1775, 1754, 1658, $1593 \mathrm{~cm}^{-1}$. Anal calcd. for $\mathrm{C}_{18} \mathrm{H}_{15} \mathrm{FN}_{4} \mathrm{O}_{2} \mathrm{~S}_{2}: \mathrm{C} 53.72$, H 3.76, N 13.92; Found: C 53.53, H 3.88, N 13.91.

$\mathrm{N}$-((4-((4-oxo-2-thioxoimidazolidin-5-yl)methyl)phenyl)carbamothioyl)-4-chlorobenzamide 9c little yellow solid, yield 58\%, m.p. $240-242{ }^{\circ} \mathrm{C},{ }^{1} \mathrm{H}$ NMR (DMSO-d 6 , $300 \mathrm{MHz}$ ) $\delta: 12.52$ (s, 1H, NH), $11.64(\mathrm{~s}, 1 \mathrm{H}, \mathrm{NH}), 11.50(\mathrm{~s}, 1 \mathrm{H}, \mathrm{NH}), 10.10(\mathrm{~s}, 1 \mathrm{H}, \mathrm{NH}), 8.00 \sim 7.97$ (m, 2H, ArH), 7.67 7.60 (m, 4H, ArH), 7.24 7.21 (m, 2H, ArH), 4.60-4.56 (m, 1H, CH), 3.01-2.96 (m, 2H, $\left.\mathrm{CH}_{2}\right)$; IR (KBr) v: 3158, 3077, 1776, 1732, 1617, $1597 \mathrm{~cm}^{-1}$. Anal calcd. for $\mathrm{C}_{18} \mathrm{H}_{15} \mathrm{ClN}_{4} \mathrm{O}_{2} \mathrm{~S}_{2}: \mathrm{C} 51.61, \mathrm{H} \mathrm{3.61,} \mathrm{N} \mathrm{13.37;}$ Found: C 51.46, H 3.72, N 13.47.

$N$-((4-((4-oxo-2-thioxoimidazolidin-5-yl)methyl)phenyl)carbamothioyl)-4-methylbenzamide 9d little yellow solid, yield 70\%, m.p. $237-238{ }^{\circ} \mathrm{C},{ }^{1} \mathrm{H}$ NMR (DMSO-d 6 , $\left.300 \mathrm{MHz}\right) \delta: 12.68$ (s, 1H, NH), $11.51(\mathrm{~s}, 1 \mathrm{H}, \mathrm{NH}), 11.43(\mathrm{~s}, 1 \mathrm{H}, \mathrm{NH}), 10.10(\mathrm{~s}, 1 \mathrm{H}, \mathrm{NH}), 7.92 \sim 7.89(\mathrm{~m}, 2 \mathrm{H}, \operatorname{ArH}), 7.67 \sim 7.64(\mathrm{~m}, 2 \mathrm{H}$, ArH), 7.36 7.33 (m, 2H, ArH), 7.24 7.21 (m, 2H, ArH), 4.59-4.56 (m, 1H, CH), 3.01-2.95 (m, 2H, $\left.\mathrm{CH}_{2}\right), 2.40\left(\mathrm{~s}, 3 \mathrm{H}, \mathrm{CH}_{3}\right)$; IR ( $\left.\mathrm{KBr}\right)$ v: 3284, 3047, 1773, 1752, 1655, $1596 \mathrm{~cm}^{-1}$. Anal calcd. for $\mathrm{C}_{19} \mathrm{H}_{18} \mathrm{~N}_{4} \mathrm{O}_{2} \mathrm{~S}_{2}$ : C 57.27, H 4.55, N 14.06; Found: C 57.20, H 4.65, N 14.11.

$N$-((4-((4-oxo-2-thioxoimidazolidin-5-yl)methyl)phenyl)carbamothioyl)-4-methoxybenzamide 9e little yellow solid, yield 46\%, m.p. $232-234{ }^{\circ} \mathrm{C},{ }^{1} \mathrm{H}$ NMR (DMSO-d 6 , $\left.300 \mathrm{MHz}\right) \delta: 12.74$ (s, 1H, NH), $11.50(\mathrm{~s}, 1 \mathrm{H}, \mathrm{NH}), 11.35$ (s, 1H, NH), $10.10(\mathrm{~s}, 1 \mathrm{H}, \mathrm{NH}), 8.03 \sim 8.00(\mathrm{~m}, 2 \mathrm{H}, \operatorname{ArH}), 7.67 \sim 7.64(\mathrm{~m}, 2 \mathrm{H}$, ArH), 7.23 7.20 (m, 2H, ArH), 7.08 7.05 (m, 2H, ArH), 4.59-4.56 (m, 1H, CH), $3.86\left(\mathrm{~s}, 3 \mathrm{H}, \mathrm{OCH}_{3}\right)$, 3.01-2.96 (m, 2H, $\left.\mathrm{CH}_{2}\right)$; IR (KBr) v: 3306, 3051, 1774, 1752, 1668, $1596 \mathrm{~cm}^{-1}$. Anal calcd. for $\mathrm{C}_{19} \mathrm{H}_{18} \mathrm{~N}_{4} \mathrm{O}_{3} \mathrm{~S}_{2}$ : C 55.05, H 4.38, N 13.52; Found: C 54.82, H 4.45, N 13.49. 
$\mathrm{N}$-((4-((4-oxo-2-thioxoimidazolidin-5-yl)methyl)phenyl)carbamothioyl)-4-nitrobenzamide 9f little yellow solid, yield 85\%, m.p. $244-245{ }^{\circ} \mathrm{C},{ }^{1} \mathrm{H}$ NMR (DMSO-d 6 , $300 \mathrm{MHz}$ ) $\delta: 12.40$ (s, 1H, NH), $11.91(\mathrm{~s}, 1 \mathrm{H}, \mathrm{NH}), 11.50$ (s, 1H, NH), 10.10 (s, 1H, NH), 8.36 8.33 (m, 2H, ArH), 8.18 8.15 (m, 2H, ArH), 7.68 7.64 (m, 2H, ArH), 7.25 7.21 (m, 2H, ArH), 4.60-4.56 (m, 1H, CH), 3.05-2.97 (m, 2H, $\mathrm{CH}_{2}$ ); IR (KBr) v: 3202, 3060, 1775, 1746, 1672, $1594 \mathrm{~cm}^{-1}$. Anal calcd. for $\mathrm{C}_{18} \mathrm{H}_{15} \mathrm{~N}_{5} \mathrm{O}_{4} \mathrm{~S}_{2}$ : C 50.34, H 3.52, N 16.31; Found: C 50.18, H 3.67, N 16.24.

$\mathrm{N}$-((4-((4-oxo-2-thioxoimidazolidin-5-yl)methyl)phenyl)carbamothioyl)-2-methylbenzamide $\quad \mathbf{9 g}$ little yellow solid, yield 63\%, m.p. $220-222{ }^{\circ} \mathrm{C},{ }^{1} \mathrm{H}$ NMR (DMSO-d $\left.6,300 \mathrm{MHz}\right) \delta: 12.58$ (s, 1H, NH), $11.68(\mathrm{~s}, 1 \mathrm{H}, \mathrm{NH}), 11.50(\mathrm{~s}, 1 \mathrm{H}, \mathrm{NH}), 10.10(\mathrm{~s}, 1 \mathrm{H}, \mathrm{NH}), 7.70 \sim 7.66(\mathrm{~m}, 2 \mathrm{H}, \mathrm{ArH}), 7.51 \sim 7.41(\mathrm{~m}, 2 \mathrm{H}$, ArH), 7.32 7.27 (m, 2H, ArH), 7.24 7.20 (m, 2H, ArH), 4.59-4.56 (m, 1H, CH), 3.02-2.95 (m, 2H, $\left.\mathrm{CH}_{2}\right), 2.42\left(\mathrm{~s}, 3 \mathrm{H}, \mathrm{CH}_{3}\right)$; IR (KBr) v: 3187, 3094, 1774, 1745, 1676, $1587 \mathrm{~cm}^{-1}$. Anal calcd. for $\mathrm{C}_{19} \mathrm{H}_{18} \mathrm{~N}_{4} \mathrm{O}_{2} \mathrm{~S}_{2}$ : C 57.27, H 4.55, N 14.06; Found: C 57.14, H 4.62, N 14.35.

$N$-((4-((4-oxo-2-thioxoimidazolidin-5-yl)methyl)phenyl)carbamothioyl)-2-chlorobenzamide $\mathbf{9 h}$ little yellow solid, yield 53\%, m.p. $216-218{ }^{\circ} \mathrm{C},{ }^{1} \mathrm{H}$ NMR (DMSO-d 6 , $\left.300 \mathrm{MHz}\right) \delta: 12.38$ (s, 1H, NH), 11.98 (s, 1H, NH), 11.51 (s, 1H, NH), 10.10 (s, 1H, NH), 7.68 7.43 (m, 6H, ArH), 7.24 7.21 (m, 2H, ArH), 4.60-4.56 (m, 1H, CH), 3.02-2.96 (m, 2H, $\left.\mathrm{CH}_{2}\right)$; IR (KBr) v: 3224, 3062, 1776, 1745, 1685, $1593 \mathrm{~cm}^{-1}$. Anal calcd. for $\mathrm{C}_{18} \mathrm{H}_{15} \mathrm{ClN}_{4} \mathrm{O}_{2} \mathrm{~S}_{2}$ : C 51.61, H 3.61, N 13.37; Found: C 51.44, H 3.70, N 13.36.

$N$-((4-((4-oxo-2-thioxoimidazolidin-5-yl)methyl)phenyl)carbamothioyl)-2-methoxybenzamide 9i little yellow solid, yield 69\%, m.p. $244-246{ }^{\circ} \mathrm{C},{ }^{1} \mathrm{H}$ NMR (DMSO-d $\left.6,300 \mathrm{MHz}\right) \delta: 12.58$ (s, 1H, NH), 11.50 (s, 1H, NH), 11.20 (s, 1H, NH), 10.09 (s, 1H, NH), 7.94 7.91 (m, 1H, ArH), 7.70 7.64 (m, 3H, ArH), 7.31 7.15 (m, 4H, ArH), 4.59-4.56 (m, 1H, CH), 4.01 (s, 3H, $\left.\mathrm{OCH}_{3}\right), 3.02-2.94$ (m, 2H, $\left.\mathrm{CH}_{2}\right)$; IR (KBr) v: 3159, 3084, 1775, 1752, 1651, $1595 \mathrm{~cm}^{-1}$. Anal calcd. for $\mathrm{C}_{19} \mathrm{H}_{18} \mathrm{~N}_{4} \mathrm{O}_{3} \mathrm{~S}_{2}$ : C 55.05, $\mathrm{H} 4.38$, N 13.52; Found: C 54.92, H 4.47, N 13.63.

$N$-((4-((4-oxo-2-thioxoimidazolidin-5-yl)methyl)phenyl)carbamothioyl)-2-nitrobenzamide 9j little yellow solid, yield 54\%, m.p. 202-204 ${ }^{\circ} \mathrm{C},{ }^{1} \mathrm{H}$ NMR (DMSO-d 6 , $300 \mathrm{MHz}$ ) $\delta: 12.29$ (s, 1H, NH), $12.12(\mathrm{~s}, 1 \mathrm{H}, \mathrm{NH}), 11.51(\mathrm{~s}, 1 \mathrm{H}, \mathrm{NH}), 10.11(\mathrm{~s}, 1 \mathrm{H}, \mathrm{NH}), 8.24 \sim 8.21(\mathrm{~m}, 1 \mathrm{H}, \mathrm{ArH}), 7.94 \sim 7.88(\mathrm{~m}, 1 \mathrm{H}$, ArH), 7.81 7.66 (m, 4H, ArH), 7.25 7.22 (m, 2H, ArH), 4.61-4.56 (m, 1H, CH), 3.02-2.96 (m, 2H, $\mathrm{CH}_{2}$ ); IR (KBr) v: 3166, 3030, 1775, 1740, 1693, $1598 \mathrm{~cm}^{-1}$. Anal calcd. for $\mathrm{C}_{18} \mathrm{H}_{15} \mathrm{~N}_{5} \mathrm{O}_{4} \mathrm{~S}_{2} \cdot 1 / 3 \mathrm{H}_{2} \mathrm{O}: \mathrm{C}$ 49.64, H 3.63, N 16.08; Found: C 49.67, H 3.68, N 16.19.

$N$-((4-((4-oxo-2-thioxoimidazolidin-5-yl)methyl)phenyl)carbamothioyl)-3-methylbenzamide $\quad 9 k$ little yellow solid, yield 88\%, m.p. $234-236{ }^{\circ} \mathrm{C},{ }^{1} \mathrm{H}$ NMR (DMSO-d 6 , $300 \mathrm{MHz}$ ) $\delta: 12.65$ (s, 1H, NH), $11.50(\mathrm{~s}, 1 \mathrm{H}, \mathrm{NH}), 11.47(\mathrm{~s}, 1 \mathrm{H}, \mathrm{NH}), 10.10(\mathrm{~s}, 1 \mathrm{H}, \mathrm{NH}), 7.83 \sim 7.65(\mathrm{~m}, 4 \mathrm{H}, \mathrm{ArH}), 7.49 \sim 7.40(\mathrm{~m}, 2 \mathrm{H}$, ArH), 7.24 7.21 (m, 2H, ArH), 4.60-4.56 (m, 1H, CH), 3.01-2.94 (m, 2H, $\left.\mathrm{CH}_{2}\right), 2.39$ (s, 3H, $\left.\mathrm{CH}_{3}\right)$; IR (KBr) v: 3176, 3051, 1776, 1751, 1657, $1597 \mathrm{~cm}^{-1}$. Anal calcd. for $\mathrm{C}_{19} \mathrm{H}_{18} \mathrm{~N}_{4} \mathrm{O}_{2} \mathrm{~S}_{2}$ : C 56.42, $\mathrm{H} 4.65$, N 13.85; Found: C 56.50, H 4.63, N 14.11.

$N$-((4-((4-oxo-2-thioxoimidazolidin-5-yl)methyl)phenyl)carbamothioyl)-3-nitrobenzamide 9l little yellow solid, yield 73\%, m.p. $242-243{ }^{\circ} \mathrm{C},{ }^{1} \mathrm{H}$ NMR (DMSO-d $6,300 \mathrm{MHz}$ ) $\delta: 12.43$ (s, 1H, NH), $11.97(\mathrm{~s}, 1 \mathrm{H}, \mathrm{NH}), 11.51(\mathrm{~s}, 1 \mathrm{H}, \mathrm{NH}), 10.10(\mathrm{~s}, 1 \mathrm{H}, \mathrm{NH}), 8.78 \sim 8.76(\mathrm{~m}, 1 \mathrm{H}, \operatorname{ArH}), 8.50 \sim 8.47(\mathrm{~m}, 1 \mathrm{H}$, $\operatorname{ArH}), 8.38 \sim 8.35$ (m, 1H, ArH), 7.86 7.81 (m, 1H, ArH), 7.68 7.65 (m, 2H, ArH), 7.25 7.22 (m, 2H, ArH), 4.60-4.56 (m, 1H, CH), 3.02-2.96 (m, 2H, $\left.\mathrm{CH}_{2}\right)$; IR (KBr) v: 3172, 3090, 1774, 1738, 1692, $1604 \mathrm{~cm}^{-1}$. Anal calcd. for $\mathrm{C}_{18} \mathrm{H}_{15} \mathrm{~N}_{5} \mathrm{O}_{4} \mathrm{~S}_{2}$ : C 50.34, H 3.52, N 16.31; Found: C 49.94, H 3.68, N 16.26. 
$N$-((4-((4-oxo-2-thioxoimidazolidin-5-yl)methyl)phenyl)carbamothioyl)thiophene-2-carboxamide 9m little yellow solid, yield 68\%, m.p. $242-244{ }^{\circ} \mathrm{C},{ }^{1} \mathrm{H}$ NMR (DMSO-d $\left.6,300 \mathrm{MHz}\right) \delta: 12.48(\mathrm{~s}, 1 \mathrm{H}$, $\mathrm{NH}), 11.58(\mathrm{~s}, 1 \mathrm{H}, \mathrm{NH}), 11.51$ (s, 1H, NH), 10.10 (s, 1H, NH), 8.39-8.37 (m, 1H, ThH), 8.06-8.04 (m, 1H, ThH), 7.66 7.63 (m, 2H, ArH), 7.27 7.20 (m, 3H, ArH + ThH), 4.60-4.55 (m, 1H, CH), 3.01-2.95 (m, 2H, $\left.\mathrm{CH}_{2}\right)$; IR (KBr) v: 3176, 3107, 1776, 1737, 1668, $1599 \mathrm{~cm}^{-1}$. Anal calcd. for $\mathrm{C}_{16} \mathrm{H}_{14} \mathrm{~N}_{4} \mathrm{O}_{2} \mathrm{~S}_{3}$ : C 49.21, H 3.61, N 14.35; Found: C 49.16, H 3.72, N 14.52.

\subsection{Bioassay of Herbicidal and Fungicidal Activity}

The preliminary herbicidal activities of compounds 7-9 against B. campestris and E. crus-galli were assayed using the protocols in the references [11-13]. The preliminary fungicidal activities of compounds 7-9 against $F$. oxysporurm, A. solani, B. berengeriana, $C$. arachidcola and $F$. graminearum were evaluated using methods in the references $[7,10]$ by the mycelium growth rate test [38]. The culture was incubated at $25 \pm 0.5^{\circ} \mathrm{C}$. Three replicates were performed and the mean measurements were calculated from the three replicates.

The greenhouse test was performed using the procedures in reference [39] according to the pre-emergence and post-emergence applications. The formulations were sprayed before the seedlings were planted in a pot or the formulations were sprayed during one to two-leaf appeared after the seedlings were planted in a pot. Then they were kept in the greenhouse to observe the root and stem growth of the plants in three weeks, and the inhibition rates of compounds were obtained comparison the fresh plant weights with the blank control. Three replicates were performed and the mean measurements were calculated from the three replicates.

\section{Conclusions}

The novel acylthiourea derivatives with hydantoin or thiohydantoin were synthesized in moderate to excellent yields using 5-(4-aminophenyl)- and 5-(4-aminobenzyl)-hydantoin or 5-(4-aminobenzyl)thiohydantoin as raw materials and characterized by IR, ${ }^{1} \mathrm{H}$ NMR spectra and elementary analysis. The preliminary bioassay showed that these compounds exhibit some herbicidal selectivity with the $91 \%$, $94 \%$ and $87 \%$ inhibition rates of $7 \mathbf{1}, \mathbf{8 0}$ and $\mathbf{8 p}$ against $B$. campestris, and $100 \%, 100 \%$ and $95 \%$ efficacy against $B$. campestris in a greenhouse test, respectively. Compounds $7 \mathbf{a}, 7 \mathbf{b}, \mathbf{7 c}$ and $\mathbf{7 d}$ exhibited $74 \%, 79 \%, 79 \%$ and $71 \%$ inhibition rates against $F$. oxysporum, respectively.

\section{Acknowledgments}

This project was founded by National Natural Science Foundation of China (Nos. 21172254 and 20772150), the 12th Five-year National Key Technologies R \& D Program of China (No. 2011BAE06B03) and National Key Laboratory of Elemento-Organic Chemistry in Nankai University (201003).

\section{Conflicts of Interest}

The authors declare no conflict of interest. 


\section{References}

1. Li, K.; Shi, D.Q. Synthesis and herbicidal activity of 3-aryl-1-[2-(aryloxy)propanoyl]imidazolidine-2,4-diones. J. Heterocycl. Chem. 2009, 46, 544-547.

2. Thenmozhiyal, J.C.; Wong, P.T.-H.; Chui, W.K. Anticonvulsant activity of phenylmethylene-hydantoins: A structure-activity relationship study. J. Med. Chem. 2004, 47, $1527-1535$.

3. Elokdah, H.; Abou-Gharbia, M.; Hennan, J.K.; Mcfarlane, G.; Mugford, C.P.; Krishnamurthy, G.; Crandall, D.L. Tiplaxtinin, a novel orally efficacious inhibitor of plasminogen activator inhibitor-1: Design, synthesis, and preclinical characterization. J. Med. Chem. 2004, 47, 3491-3494.

4. Brady, S.F.; Bauer, J.D.; Clarke-Pearson, M.F.; Daniels, R. Natural products from isnA-containing biosynthetic gene clusters recovered from the genomes of cultured and uncultured bacteria. J. Am. Chem. Soc. 2007, 129, 12102-12103.

5. Nakajima, M.; Itoi, K.; Takamatsu, Y.; Kinoshita, T.; Okazaki, T.; Kawakubo, K.; Shindo, M.; Honma, T.; Tohjigamori, M.; Haneishi, T. Hydantocidin: A new compound with herbicidal activity from Streptomyes hygroscopicus. J. Antibiot. 1991, 44, 293-300.

6. Zhao, B.G.; Du, H.F.; Shi, Y.A. A Cu(I)-catalyzed C-H $\alpha$-Amination of esters. Direct synthesis of hydantoins. J. Am. Chem. Soc. 2008, 130, 7220-7221.

7. Lei, J.P.; Han, J.T.; Xu, Z.H.; Dong, H.B.; Wang, M.A. Synthesis and fungicidal activity of 5-cyclohexylidene-2-aminoimidazolin-4-one derivatives. Chin. J. Org. Chem. 2012, 32, 1993-1998.

8. Kiec-Kononowicz, K.; Szymanska, E. Antimycobacterial activity of 5-arylidene derivatives of hydantoin. Farmaco 2002, 57, 909-916.

9. Kiec-Kononowicz, K.; Karolak-Wojicechowska, J.; Muller, C.E.; Schumacher, B.; Pekela, E.; Szymanska, E. Imidazo-thiazine, -diazinone and -diazepinone derivatives. Synthesis, structure and benzodiazepine receptor binding. Eur. J. Med. Chem. 2001, 36, 407-419.

10. Han, J.T.; Dong, H.B.; Xu, Z.H.; Lei, J.P.; Wang, M.A. Facile synthesis of 5-arylidene thiohydantoin by sequential sulfonylation/desulfination reaction. Int. J. Mol. Sci. 2013, 14, 12484-12495.

11. Han, J.T.; Wang, J.M.; Dong, H.B.; Xu, Z.H.; Liu, B.; Wang, M.A. Synthesis and biological activity of novel phosphoramidate with hydantoin. Chin. J. Org. Chem. 2013, 33, 596-601.

12. Xu, Z.H.; Wang, J.M.; Han, J.T.; Liu, B.; Wang, M.A. Synthesis and biological activity of novel (thio)phosphates with hydantoin. Chin. J. Org. Chem. 2012, 32, 2134-2140.

13. Han, J.T.; Dong, H.B.; Wang, J.M.; Lei, J.P.; Wang, M.A.; Fang, J.X. Synthesis and herbicidal activity of 5-(4-hydroxybenzyl)-2-thioxoimidazolidin-4-one esters. Molecules 2011, 16, 2833-2845.

14. Zhang, J.F.; Liu, C.; Ma, Y.; Wang, B.L.; Xiong, L.X.; Yu, S.J.; Li, Z.M. Synthesis of novel 3-chloropyridin-2-yl-pyrazole derivatives and their insecticidal, fungicidal activities and QSAR Study. Lett. Drug Des. Discov. 2013, 10, 497-506.

15. Zhang, J.F.; Xu, J.Y.; Wang, B.L.; Li,Y.X.; Xiong, L.X.; Li, Y.Q.; Ma, Y.; Li, Z.M. Synthesis and insecticidal activities of novel anthranilic diamides containing acylthiourea and acylurea. J. Agric. Food Chem. 2012, 60, 7565-7572. 
16. Limban, C.; Marutescu, L.; Chifiriuc, M.C. Synthesis, spectroscopic properties and antipatho-genic activity of new thiourea derivatives. Molecules 2011, 16, 7593-7607.

17. Limban, C.; Missir, A.V.; Chirita, I.C.; Nitulescu, G.M.; Caproiu, M.T.; Chifiriuc, M.C.; Israil, A.M. Synthesis and antimicrobial properties of new 2-((4-ethylphenoxy)methyl)benzoylthioureas. Chem. Pap. 2011, 65, 60-69.

18. Rao, X.P.; Wu, Y.; Song, Z.Q.; Shang, S.B.; Wang, Z.D. Synthesis and antitumor activities of unsymmetrically disubstitutedacylthioureas fused with hydrophenanthrene structure. Med. Chem. Res. 2011, 20, 333-338.

19. Sun, R.F.; Zhang, Y.L.; Chen, L.; Li, Y.Q.; Li, Q.S.; Song, H.B.; Huang, R.Q.; Bi, F.C.; Wang, Q.M. Design, synthesis, bioactivity, and structure-activity relationship (SAR) studies of novel benzoylphenylureas containing oxime ether group. J. Agric. Food Chem. 2008, 56, 11376-11391.

20. Hallur, G.; Jimeno, A.; Dalrymple, S.; Zhu, T.; Jung, M.K.; Hidalgo, M.; Isaacs, J.T.; Sukumar, S.; Hamel, E.; Khan, S.R. Benzoylphenylurea sulfur analogues with potent antitumor activity. J. Med. Chem. 2006, 49, 2357-2360.

21. Sun, C.W.; Huang, H.; Feng, M.Q.; Shi, X.L.; Zhang, X.D.; Zhou, P. A novel class of potent influenza virus inhibitors: Polysubstitutedacylthiourea and its fused heterocycle derivatives. Bioorg. Med. Chem. Lett. 2006, 16, 162-166.

22. Junejo, J.; Ghosh, S.K.; Shaikh, M.; Gahtori, P.; Singh, U.P. Facile synthesis, antibacterial activity and molecular properties prediction of some new 1,3-dihydroimidazol-2-thione derivatives. Lett. Drug Des. Discov. 2011, 8, 763-768.

23. Aamer, S.; Mahira, B. Synthesis and bioactivity of some new 1-tolyl-3-aryl-4-Methyl imidazole-2-thiones. Med. Chem. Res. 2007, 16, 143-154.

24. Wang, X.C.; Wang, F.; Quan, Z.J.; Wang, M.G.; Li, Z. An efficient and clean synthesis of 1-aroyl-3-aryl-4-substituted imidazole-2-thiones in water. J. Chem. Res. 2005, 11, 689-690.

25. Zeng, R.S.; Zou, J.P.; Zhi, S.J.; Chen, J.; Shen, Q. Novel synthesis of 1-aroyl-3-aryl-4-substituted imidazole-2-thiones. Org. Lett. 2003, 5, 1657-659.

26. Majid, M.H.; Setareh, M. An efficient synthesis of thiazol-2-imine derivatives via a one-pot, three-component reaction. Tetrahedron Lett. 2012, 53, 392-394.

27. Wang, L.; Pan, X.Q.; Rao, W.D.; Zou, J.P. Synthesis of 1,3-disubstituted-2-thioxo-4imidazolidinones. Chin. J. Org. Chem. 2011, 31, 1939-1942.

28. Aamer, S.; Uzma, S.; Abdul, H.; Faiza, K. Synthesis and antimicrobial activity of some novel 2-(substituted fluorobenzoylimino)-3-(substituted fluorophenyl)-4-methyl-1,3-thiazolines. J. Fluorine Chem. 2010, 131, 333-339.

29. Murru, S.; Singh, C.B.; Kavala, V.; Patel, B.K. A convenient one-pot synthesis of thiazol-2imines: Application in the construction of pifithrin analogues. Tetrahedron 2008, 64, 1931-1942.

30. Aamer, S.; Sabah, Z.; Michael, B. Synthesis and crystal structure of some novel 2-aroylimino-3aryl-4-phenyl-1,3-thiazolines. Synth. Commun. 2008, 38, 2185-2199.

31. Singh, C.B.; Siva, M.; Veerababurao, K.; Bhisma, K.P. 3-Aryl-1-benzoylthioureas with $\alpha$-bromoketones in water form 2- $N$-benzoyl-3-arylthiazol-2(3H)-imines, not 3-aryl-1-benzoylimidazoline-2-thiones. J. Chem. Res. 2007, 3, 136-137. 
32. Aamer, S.; Madood, P. Synthesis, crystal structure of Some new 2-(4-methylbenzoyl-imino)-3aryl-4-methyl-1,3-thiazolines. J. Heterocycl. Chem. 2006, 43, 1027-1030.

33. Singh, C.B.; Siva, M.; Veerababurao, K.; Bhisma, K.P. It is "thiazolidene-2-imine" and not imi-dazole-2-thione as the reaction product of 1-benzoyl-3-phenylthiourea with $\mathrm{Br}_{2}$ /enolizable ketone. Org. Lett. 2006, 23, 5397-5399.

34. Walters, E.W.; Lee, S.F.; Niderman, T.; Bemasconi, P.; Subramanian, M.V.; Siehl, D.L. Adenylosuccinate synthetase from maize. Purification, properties, and mechanism of inhibition by 5'-phosphohydantocidin. Plant Physiol. 1997, 114, 549-555.

35. Cseke, C.; Gerwick, B.C.; Crouse, G.D.; Murdoch, M.G.; Green, S.B.; Heim, D.R. 2-Phosphohydantocidin: the in vivo adenylosuccinate synthetase inhibitor responsible for hydantocidin phytotoxicity. Pestic. Biochem. Physiol. 1996, 55, 210-217.

36. Fonne-Pfister, R.; Chemla, P.; Ward, E.; Girardet, M.; Kreuz, K.E.; Hinzatko, R.B.; Fromm, H.; Schar, H.P.; Grutter, M.G.; Cowan-Jacob, S.W. The mode of action and the structure of a herbicide in complex with its target: Binding of activated hydantocidin to the feedback regulation site of adenylosuccinate synthetase. Proc. Natl. Acad. Sci. USA 1996, 93, 9431-9436.

37. Wang, J.M.; Xu, Z.H.; Han, J.T.; Dong, H.B.; Liu, B.; Wang, M.A. Synthesis and biological activity of novel phosphoramide with hydantoin. Chin. J. Org. Chem. 2013, doi:10.6023/cjoc201305023.

38. Chen, N.C. The Bioassay Technologies for Pesticides; Beijing Agricultural University Press: Beijing, China, 1991; pp. 161-162.

39. Chen, W.Y. The Research \& Development of New Pesticide-Methods \& Progress; Chemical Industry Press: Beijing, China, 2007; pp. 132-134.

(C) 2013 by the authors; licensee MDPI, Basel, Switzerland. This article is an open access article distributed under the terms and conditions of the Creative Commons Attribution license (http://creativecommons.org/licenses/by/3.0/). 